\title{
Does emergency medicine length of stay predict trauma outcomes at a Level 1 Trauma Center?
}

\author{
Erin Powers Kinney ${ }^{1}$, Kamal Gursahani ${ }^{1}$, Eric Armbrecht ${ }^{2}$, Preeti Dalawari ${ }^{* 1}$ \\ ${ }^{1}$ Division of Emergency Medicine, Saint Louis University School of Medicine, Saint Louis, Missouri, United States \\ ${ }^{2}$ Center for Outcomes Research, Saint Louis University, Saint Louis, Missouri, United States
}

Received: April 7, 2015

DOI: $10.5430 /$ jha.v4n5p1
Accepted: May 15, 2015

Online Published: May 28, 2015

URL: http://dx.doi.org/10.5430/jha.v4n5p1

\begin{abstract}
Objective: Previous studies looking at emergency department (ED) crowding and delays of care on outcome measures for certain medical and surgical patients excluded trauma patients. The objectives of this study were to assess the relationship of trauma patients' ED length of stay (EDLOS) on hospital length of stay (HLOS) and on mortality; and to examine the association of ED and hospital capacity on EDLOS.

Methods: This was a retrospective database review of Level 1 and 2 trauma patients at a single site Level 1 Trauma Center in the Midwest over a one year period. Out of a sample of 1,492, there were 1,207 patients in the analysis after exclusions. The main outcome was the difference in hospital mortality by EDLOS group (short was less than 4 hours $v s$. long, greater than 4 hours). HLOS was compared by EDLOS group, stratified by Trauma Injury Severity Score (TRISS) category $(<0.5,0.51-0.89,>0.9)$ to describe the association between ED and hospital capacity on EDLOS.

Results: There was no significant difference in mortality by EDLOS (4.8\% short and $4 \%$ long, $p=.5$ ). There was no significant difference in HLOS between EDLOS, when adjusted for TRISS. ED census did not affect EDLOS ( $p=.59)$, however; EDLOS was longer when the percentage of staffed hospital beds available was lower $(p<.001)$.

Conclusions: While hospital overcrowding did increase EDLOS, there was no association between EDLOS and mortality or HLOS in leveled trauma patients at this institution.
\end{abstract}

Key Words: Emergency department, Length of stay, Mortality, Trauma, Crowding

\section{INTRODUCTION}

The Centers for Disease Control (CDC) reported 129.8 million emergency department (ED) visits in 2010. This number has increased from 42 million visits in 1960. In comparison, there were 37.9 million injury related visits in $2010 .^{[1,2]}$ While the number of ED visits has been increasing exponentially, the number of EDs across the country has decreased. ${ }^{[3]}$ ED overcrowding has been a pressing issue, with existing studies postulating associations between lengths of stay in the ED and quality outcome measures, such as timely an- tibiotic delivery for pneumonia or intervention for MI. ${ }^{[4-6]}$ Some literature suggests that ED length of stay (EDLOS) increases mortality and inpatient length of stay ${ }^{[6-8]}$ Recently, a study targeting trauma patients concluded that EDLOS is an independent predictor of trauma patient outcomes. ${ }^{[8]}$

\section{Goal of this investigation}

The objective of this study is to examine the relationships of trauma patients' EDLOS on hospital length of stay (HLOS), and on mortality. The primary hypothesis is that length of

\footnotetext{
*Correspondence: Preeti Dalawari; Email: pdalawar@slu.edu; Address: School of Medicine, Division of Emergency Medicine, 3635 Vista at Grand Avenue, First Floor Desloge Towers, Saint Louis, Missouri 63110, United States. 
stay greater than four hours is neither associated with longer HLOS nor higher mortality in trauma patients. The second objective of the study is to examine the relationship between EDLOS in trauma patients and ED and hospital capacity at the time of trauma activation.

\section{MeTHODS}

\subsection{Study design}

This is an exempt Institutional Review Board approved retrospective database review at a Level 1 Trauma Center in the Midwest. Data was obtained via report from the single site data collection and reporting system (Collector Registry), which contains de-identified information on all leveled trauma patients. Data from this registry is used to report to the National Trauma Database and the Trauma Quality Improvement Program. Only Level 1 and Level 2 activated trauma patients from July 1, 2010 to June 30, 2011 were included in this study.

\subsection{Setting}

The study hospital has a twenty bed ED with two additional trauma bays. In the triage area, mid-level providers often see and disposition lower acuity patients. The triage area is not included in the 22 beds. The ED census was 36,552 for the study timeframe. Admission rates were $30 \%$. The average ED LOS for all patients was 244 minutes respectively.

\subsection{Trauma activation criteria}

Trauma patients are given a Level 1, 2, or 3 designation based on criteria determined by a hospital committee of nurses, administrators and physicians involved in the care of trauma patients.

Table 1. Trauma triage criteria at this institution

\begin{tabular}{|c|c|}
\hline Level & Varieties \\
\hline \multirow{6}{*}{ Level 1} & Confirmed blood pressure $<90$ systolic at any time \\
\hline & Gunshot wounds to the neck, chest, or abdomen \\
\hline & Glasgow Coma Scale $<8$ with mechanism attributed to trauma \\
\hline & Transfer patients from other hospitals receiving blood to maintain vital signs \\
\hline & Respiratory compromise/obstrution and/or intubation in a patient who is not transferred from another facility \\
\hline & Emergency physician’s discretion \\
\hline \multirow{15}{*}{ Level 2} & Intubated patient who has been stabilized and transferred from another facility \\
\hline & Penetrating injury to the head, neck, torso, or extremities proximal to the elbow or knee \\
\hline & Open and depressed skull fracures \\
\hline & Flail chest or multiple rib fractures \\
\hline & Traumatic amputation of extremity \\
\hline & Two or more long bone fractures \\
\hline & Pelvic fractures \\
\hline & Degloving/crush injuries/mangeld extremity \\
\hline & High Risk Auto crash: \\
\hline & Intrusion: > 12 inches, occupant site; > 18 inches, any site \\
\hline & Ejection (parital or complete) from automobile \\
\hline & Death in the same passenger compartment \\
\hline & Auto vs pedestrian/bicylcist thrown, run over, or with significant impact (>20 mph) \\
\hline & Motorcycle crash $>20 \mathrm{mph}$ \\
\hline & Emergency physician's discretion \\
\hline
\end{tabular}

Table 1 lists the institution specific criteria for Levels 1 and 2 . Level 3 constitutes all trauma consults that were not leveled initially. The trauma team consists of an ED attending, an ED resident, a trauma attending, senior and junior trauma residents, ED nurses, a respiratory therapist, and a radiology technician.

Several validated scoring schema have been developed to predict prognosis in trauma patients. The Collector Registry records the Trauma and Injury Severity Score (TRISS) for each leveled trauma patient. TRISS, which is calculated from the Injury Severity Score (ISS) and Revised Trauma Score (RTS), predicts the probability of trauma patient survival, taking age and mechanism (blunt versus penetrating) into account. The ISS is an anatomical scoring system that provides an overall score for patients with multiple injuries. Each injury is assigned an Abbreviated Injury Scale (AIS) score (minor to un-survivable numbered 1-6) and is allocated 
to body region (head, face, chest, abdomen, extremities, and external). The highest AIS in each region and the three most injured regions are added together to produce the ISS. ISS values range from $0-75$. The RTS is a physiologic scoring system. It is scored from the first data obtained on a patient and consists of Glasgow Coma Scale, systolic blood pressure and respiratory rate. The higher the TRISS score, the greater probability of survival. Thus, the higher the TRISS score the less "sick" a patient will be compared to a low TRISS score.

\subsection{Study protocol}

A report generated from the Collector's registry included demographics, time of trauma activation, TRISS, trauma type (blunt $v s$. penetrating), admission time, admitting service, and hospital disposition and time.

Hospital capacity data at the time of trauma activation was collected from the hospital bed board. This capacity data included absolute numbers of available physical beds, staffed beds, floor beds and ICU beds at the time of the leveled trauma patients' arrivals. The percentage of total staffed hospital beds, which includes both floor and ICU were used in the analysis. ED capacity data was based on number of patients checked into the ED at the time of trauma activation.

\subsection{Definitions and measures}

EDLOS was categorized as "short" if duration was less than 4 hours, consistent with the study hospital's average EDLOS for all patients during the study timeframe. This categorization was also chosen based on the National Hospital Ambulatory Medical Care Survey published by the CDC, which reported that $71 \%$ of visits to EDs lasted four hours or less in 2010. ${ }^{[2]}$ Given the distribution of TRISS being highly skewed towards survivability (higher TRISS scores); we recoded TRISS as a categorical variable, into 3 levels (less than 0.5, between 0.51-0.89, and greater than 0.9).

The primary outcome measure examined in this study was all cause in hospital mortality. The secondary outcome measure was HLOS defined as days in the hospital from admission to discharge. Hospital and ED census data were also obtained and analyzed in an attempt to assess if hospital overcrowding contributes substantially to increased ED LOS. ${ }^{[9]}$

\subsection{Data analysis}

Those who died in the ED, were not admitted to the hospital, or left against medical advice (AMA) from the ED were excluded from mortality analysis. A $z$-test for proportions was used to assess differences in hospital mortality by EDLOS group (short vs. long). Differences in gender, age, trauma type, weekend admission, and in-patient staffing level at the time of admission (demographic variables) were compared Published by Sciedu Press by EDLOS group. Categorical demographical variables were analyzed by chi square analysis whereas continuous demographic and capacity variables were analyzed by student's $t$ test.

Analysis of variance (ANOVA) was employed to compare HLOS by EDLOS group, stratified by TRISS category $(<0.5,0.51-0.89,>0.9)$. In addition to the previous exclusions, analyses for HLOS also excluded patients who died in the hospital and those who left AMA after admission. If a patient died in the ED or left AMA from the ED, their EDLOS was artificially shortened, and they had no HLOS. If a patient left AMA or died while admitted in the hospital, the HLOS was artificially shortened. For the purposes of analysis, HLOS was also capped at 30 days to reduce outlier bias. In addition, a sensitivity analysis (using correlation coefficient) was conducted in which EDLOS was retained as a continuous variable to examine bivariate correlation with HLOS.

The influence of hospital and ED crowding on EDLOS was determined using a measure of ED capacity based on a bed count of 20. The analysis included all patients checked into (or registered into) the ED at the time of the trauma activation. In other words, an ED capacity of 1.00 means 20 patients are checked into the ED (which has a total of 20 available beds); a ED capacity of 1.25 indicates there were 25 patients checked in at the time of trauma activation. This measure has been validated for ED crowding in a previous study. ${ }^{[10]}$

A flowchart of included patients is in Figure 1. There were 1,492 Level 1 or 2 trauma activations during the study period (out of 2,507 total traumas which includes Levels 1, 2, and $3)$.

\section{Results}

As seen in Table 2, the final sample of 1,207 patients includes $24 \%$ females and the mean age was 39 (SD 17 years). The total study population had a mean EDLOS of 214 minutes (SD 130).

Approximately two-thirds of patients $(807$ of 1,207$)$ had a short EDLOS. There was no difference in the mortality rates between short (4.8\%) and long (4.0\%) EDLOS groups, $p=$ .50. Mean HLOS was approximately 1.3 days less for patients in the long EDLOS group, $p=.017$. The mean HLOS for the two groups were: short 8.6 ( $S D$ 8.8 days) versus long 7.3 (SD 8.2 days). The average TRISS scores between these two groups were similar: short $0.93(0.16)$ versus long 0.95 (0.14), $p=.11$. Overall, about $87 \%$ of patients had a high TRISS classification ( $>0.9) ; 9 \%$ were medium, and $4 \%$ were low or the least likely to survive $(<0.5)$. 


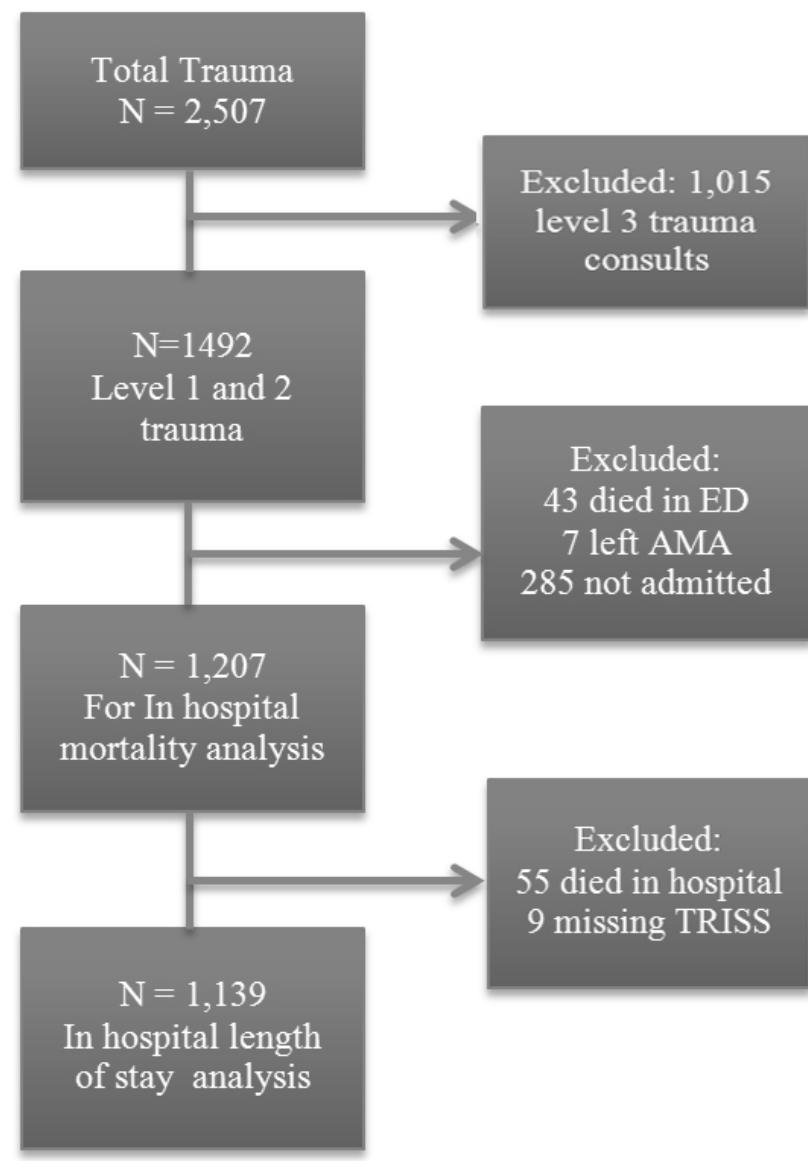

Figure 1. Eligibility flowchart

After excluding the fifty-five patients who died in the hospital and the nine with missing TRISS scores $(n=1,139)$, HLOS was compared by EDLOS group, as seen in Figure 2. The stratified analysis revealed that there is actually no association between HLOS and EDLOS after adjustment for TRISS classification (low TRISS 20.0 vs. 21.3 days; medium TRISS 15.4 vs. 14.4 days; high TRISS 7.2 vs. 6.3 days; $p=$ .49). Thus, HLOS is associated with TRISS classification, $p<.001$. In other words, severely injured patients have a longer HLOS regardless of how long they are in the ED. For example, the HLOS is 2.7 times greater for patients with low TRISS classification (more severely injured) in comparison to those with high TRISS (less injured) among patients with short EDLOS. The relative difference in HLOS is similar among patients with long EDLOS as the HLOS is 3.4 times greater for patients with low versus high TRISS classification.

A sensitivity analysis in which EDLOS was coded as a continuous variable revealed that EDLOS has a weak negative association with HLOS (correlation coefficient $=-.08$, $p=.01$ ), indicating that HLOS decreased as EDLOS increased; this finding is directionally consistent with the analysis in which EDLOS was coded as a categorical variable: short vs. long.

The association between ED crowding and EDLOS was not statistically significant. EDLOS was associated with hospital capacity. Trauma activations with short EDLOS had a mean percentage of staffed occupied floor and ICU beds of 80.0; by comparison, trauma activations with long EDLOS had a mean percentage 1.8 percentage points higher. A higher percentage of staffed floor and ICU beds indicate a greater proportion were occupied, and therefore fewer available for admission.

\section{Discussion}

Length of stay has become an important quality indicator for EDs due to its telling measure of patient flow from ED arrival to disposition. Increased EDLOS has been linked to poor patient satisfaction as well as hospital and ED overcrowding, and it may be associated with adverse clinical outcomes. ${ }^{[4,7]}$ Crowding and patient flow in the ED are of such importance, the Centers for Medicare and Medicaid Services (CMS) began collecting data on measures such as EDLOS on January $1^{\text {st }}, 2012$. As a result, much interest in finding associations among ED throughput measures and clinical outcomes has been generated.

Studies examining the relationship between critical care patients' EDLOS and quality outcomes, including cardiovascular and sepsis measures have produced mixed results. ${ }^{[1]}$ However, it is universally accepted that critically ill patients boarding in the ED use substantial resources including ED physician time. ${ }^{[12]}$

In 2007, a three-year retrospective multi-center database review concluded that EDLOS greater than six hours increased both HLOS and mortality for critical care patients. ${ }^{[7]}$ Two years later, a prospective study, including 3,918 patients, also using the six hour marker, found that experienced emergency physicians can effectively triage more critically ill surgical (trauma and emergency surgical) patients to the ICU; therefore, EDLOS for this level of case severity did not increase inpatient mortality. ${ }^{[6]}$

In June 2011, Mowery et al. published the first study specifically examining the relationship between EDLOS and trauma patient outcomes. They concluded that hospital mortality increases for each additional hour a trauma patient spends in the ED, and that $8.3 \%$ of these patients staying in the ED between four and five hours would ultimately die. It was suggested that diagnostic evaluation, procedures, and consulting services were the causes of longer EDLOS measures. ${ }^{[8]}$ Of note, they excluded any patients who were in the ED for greater than five hours from their data because they assumed lower ISS for that group. 
Table 2. Demographic and outcome variables by EDLOS

\begin{tabular}{|c|c|c|c|c|}
\hline Characteristics & Short EDLOS & Long EDLOS & Total & $p$-value \\
\hline Full Sample & $\mathrm{n}=807$ & $\mathrm{n}=400$ & $\mathrm{n}=1,207$ & \\
\hline Gender, n (\%) & & & & $<.001$ \\
\hline Female & $178(22)$ & 115 (29) & $293(24)$ & \\
\hline Male & $629(78)$ & $285(71)$ & 914 (76) & \\
\hline Age, mean $(S D)$ & $38(17)$ & $40(17)$ & 39 (17) & .081 \\
\hline Trauma Type, n (\%) & & & & $<.001$ \\
\hline Blunt & $584(72)$ & $353(88)$ & 937 (78) & \\
\hline Penetrating & $221(27)$ & $45(11)$ & $266(22)$ & \\
\hline Day of Arrival, n (\%) & & & & .478 \\
\hline Weekday & $386(48)$ & $200(50)$ & $586(49)$ & \\
\hline Weekend & $421(52)$ & $200(50)$ & $621(51)$ & \\
\hline ED Disposition, n (\%) & & & & $<.001$ \\
\hline Floor & $373(46)$ & $244(61)$ & $617(51)$ & \\
\hline ICU & 218 (27) & 129 (32) & 347 (29) & \\
\hline OR & 216 (27) & $27(7)$ & $243(20)$ & \\
\hline Mortality (\%) & 4.8 & 4.0 & 4.5 & .500 \\
\hline Hospital Capacity $^{* *}$ & & & & $<.001$ \\
\hline Mean \% & 80 & 82 & 81 & \\
\hline \multicolumn{5}{|l|}{ ED Census, mean (SD) } \\
\hline Count of patients & $39.9(16.3)$ & $41.8(17.3)$ & 40.5 (16.6) & .059 \\
\hline Capacity & $1.99(0.81)$ & $2.09(0.86)$ & $2.03(0.83)$ & .059 \\
\hline Discharged Alive & $\mathrm{n}=768$ & $\mathrm{n}=384$ & $\mathrm{n}=1,152$ & \\
\hline HLOS, mean (SD) & $8.6(8.8)$ & $7.3(8.2)$ & $8.2(8.6)$ & .017 \\
\hline TRISS, mean (SD) & $0.93(0.16)$ & $0.95(0.14)$ & $0.94(0.16)$ & .110 \\
\hline TRISS Categories, n (\%) & & & & .077 \\
\hline$>0.9$ & $649(85)$ & 343 (89) & $992(86)$ & \\
\hline $0.51-0.89$ & $76(10)$ & $25(7)$ & $101(9)$ & \\
\hline$<0.5$ & $34(4)$ & $12(3)$ & $46(4)$ & \\
\hline Missing & $9(1)$ & $4(1)$ & $13(1)$ & \\
\hline
\end{tabular}

"patient missing data from each group; ${ }^{* *}$ Mean $\%$ occupied staffed beds

Although the National Quality Forum recommended that CMS follow EDLOS indicators as early as 2008, there is still no consensus as to appropriate specific goals for these times nor the percentage of patients that must fall within these time frames. ${ }^{[13]}$ For the purposes of this study, the institution's goal of an average EDLOS of less than 240 minutes for admitted patients was used as the cut off between the short and long EDLOS groups. Using this benchmark, the analysis showed that there is no association between EDLOS and mortality in leveled trauma patients. These results challenge the findings reported by Mowery et al.

This study not only examined the relationship between EDLOS and mortality, but also EDLOS and its relationship to other quality indicators. HLOS is a measure used to follow the efficiency and cost effectiveness of patient care delivery. Prolonged HLOS may also result in a greater risk of adverse events such as venous thromboembolic disease, nosocomial infections, and medication errors. Furthermore, each additional day spent in the hospital accrues significant costs for patients and payers.

Surprisingly, the data collected in this study revealed that patients in the short EDLOS group had longer HLOS than patients in the long EDLOS group. When corrected for TRISS, the groups actually have similar HLOS. Therefore, it is actually the severity of traumatic injuries as determined by TRISS designation that influences leveled trauma patients' HLOS, not their EDLOS (see Figure 2).

While this study failed to show a strong relationship between EDLOS and HLOS and mortality in leveled trauma patients, it does confirm the findings of previous research: EDLOS is a measure of patient care flow and is therefore influenced by hospital capacity. ${ }^{[14-16]}$ Leveled trauma patients with short EDLOS arrived at times when there happened to be more 
staffed beds available in the hospital. Conversely, those with long EDLOS arrived at times in which the hospital was more full, in other words, there were less staffed beds available in which to admit them.

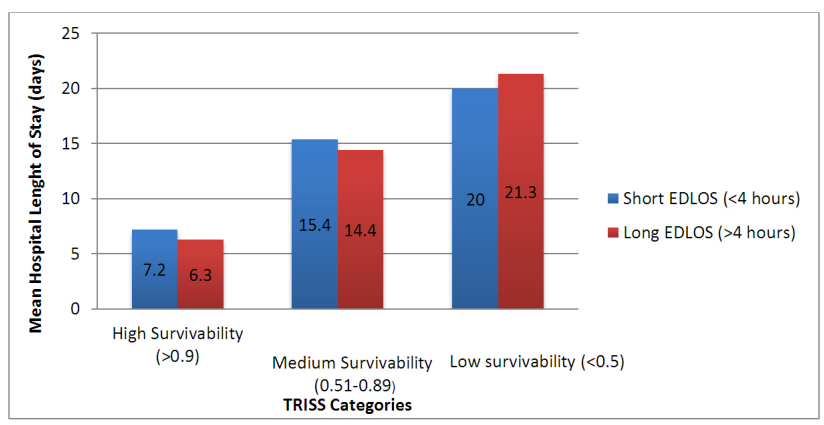

Figure 2. LOS association with TRISS $(n=1,139)$

This study also examined the relationship between EDLOS and ED capacity in leveled trauma patients. Although there is no standard measure of ED capacity to date, the total number of patients checked into the ED at time of trauma activation was used in this study. This measure includes those patients waiting to be seen. While hospital capacity had a statistically significant association with short and long EDLOS, ED capacity did not.

EDLOS in and of itself has become an accepted measure of both ED and hospital crowding. More recently, a study at Boston University looked into specific factors which increase EDLOS: nurses on duty, discharges, discharges on previous shift, resuscitation cases, admissions, ICU admissions, and hospital occupancy. Similar to this study they found that EDLOS was associated with hospital occupancy (EDLOS increased by $1 \mathrm{~min}$ for every $1 \%$ increase in hospital capacity). ${ }^{[9]}$

This study supports the notion that EDLOS is a measure associated with the efficient use of hospital resources. Reducing EDLOS requires a hospital wide effort. Clinical outcome (i.e. mortality rates and HLOS) of trauma patients were not dependent on EDLOS. EDLOS should not be used independently as a benchmark in leveled trauma patients because as this study has shown, it has no impact on outcomes. EDLOS, however, should continue to be a measure of hospital capacity and throughput as it is undeniably affected by hospital overcrowding.

\section{Limitations}

This was a single center urban Level 1 Trauma Center with a trauma team that responds to all leveled traumas. Our data will reflect our specific study environment and available resources and may not be applicable to all other ED settings.

Our patient data came from the Collector's registry, a single site database with reporting capability for state and national trauma registries. We did not conduct chart reviews. Therefore, we are unable to comment on procedures done in the $\mathrm{ED}$, number of consulting services, or complications in care that could have contributed to mortality or HLOS. These additional measures can affect ED LOS and could be further explored in future studies.

Finally, it may seem like there is an unusually high number of male trauma patients in this study. However, the National Trauma Data Bank Annual Report acknowledges that males account for $70 \%$ of all incidents up to the age of 70 , after which females predominate. ${ }^{[1]}$ With a mean age of 39 years for this study population, $76 \%$ being male is not surprising.

\section{Conclusions}

While hospital overcrowding did increase EDLOS, we found no association between EDLOS greater than four hours and mortality in leveled trauma patients. The association between shorter EDLOS and longer HLOS was clarified by TRISS score.

\section{ACKNOWLEDgements}

The authors would like to acknowledge Avni Shah for assistance with data collection.

\section{REFERENCES}

[1] Garcia TC, Bernstein AB, Bush MA. Emergency department visitors and visits: who used the emergency room in 2007? NCHC Data Brief. May 2010; 38: 1-8. http://dx.doi.org/10.1186/1 471-2261-3-9

[2] National Hospital Ambulatory Medical Care Survey: 2010 Emergency Department Summary Tables. [cited 2013 Jun 20]. Available from: http://www.cdc.gov/nchs/data/ahcd/nhamcs_ emergency/2010_ed_web_tables.pdf
[3] Pitts SR, Niska RW, Xu J, et al. National Hospital Ambulatory Medical Care Survey: 2006 emergency department summary. National Health Statistics Report. Hyattsville, MD: National Center for Health Statistics; 2008. [cited 2013 Jun 20]. Available from: http://www.cdc.gov/nchs/data/nhsr/nhsr007.pdf

[4] Carr BG, Kaye AJ, Wiebe DJ, et al. Emergency department length of stay: a major risk factor for pneumonia in intubated blunt trauma patients. J Trauma. 2007; 63(1): 9-12. PMid: 17622862. http://dx.doi.org/10.1097/TA.0b013e31805d8f6b 
[5] Pines JE, Pollack CH, Diercks DE, et al. The Association Between Emergency Department Crowding and Adverse Cardiovascular Outcomes in Patients with Chest Pain. Acad Emerg Med. 2009; 16(7): 617-625. PMid: 19549010. http://dx.doi.org/10.1111/j.1 553-2712.2009.00456.x

[6] Richardson DJ, Franklin G, Santos A, et al. Effective triage can ameliorate the deleterious effects of delayed transfer of trauma patients from the emergency department to the ICU. J Am Coll Surg. 2009; 208: 671-81. PMid: 19476813. http://dx.doi.org/10.1016/j .jamcollsurg.2008.11.018

[7] Chalfin DB, Trzeciak S, Likourezos A, et al. Impact of delayed transfer of critically ill patients from the emergency department to the intensive care unit. Crit Care Med. 2007; 35(6): 1477-83. PMid: 17440421. http://dx.doi.org/10.1097/01.CCM.0000 266585.74905.5A

[8] Mowery NT, Dougherty SD, Hildreth AN, et al. Emergency department length of stay is an independent predictor of hospital mortality in trauma activation patients. J Trauma. 2011; 70(4): 1317-25. PMid: 21817968. http://dx.doi.org/10.1097/TA.0b013e31 82175199

[9] Rathlev NK, Obendorfer D, White LF. Time series analysis of emergency department length of stay per 8-hour shift. West J Emerg Med. 2012; 13(2): 163-8. PMid: 22900106. http://dx.doi.org/10. 5811 /west jem. 2011.6743

[10] Hwang U, McCarthy ML, Aronsky D, et al. Measure of crowding in the emergency department: a systematic review. Acad Emerg Med. 2011; 18: 527-38. PMid: 21569171. http://dx.doi.org/10.11 $11 / \mathrm{j} .1553-2712.2011 .01054 . \mathrm{x}$

[11] Harris B, Bai J, Kulstad EB. Crowding does not adversely affect time to percutaneous coronary intervention for acute myocardial infarction in a community emergency department. Ann Emerg Med. 2012; 59 13-7. PMid: 21802774. http://dx.doi.org/10.1016/j.annem ergmed.2011.06.545

[12] Fromm RE, Gibbs LR, McCallum WGB, et al. Critical care in the emergency department: A time based study. Crit Care Med. 1993; 21(7): 970-6. PMid: 8319477. http://dx.doi.org/10.1097/0 0003246-199307000-00009

[13] National Voluntary Consensus Standards for Emergency Care: A Consensus Report. Washington, DC: National Quality Forum; 2009. [cited 2013 Jun 20]. Available from: http://www.qualityforum .org/Publications/2009/09/National_Voluntary_Conse nsus_Standards_for_Emergency_Care.aspx

[14] Derlet RW, Richards JR. Overcrowding in the nation's emergency departments: complex causes and disturbing effects. Ann Emerg Med. 2000; 35: 63-68. http://dx.doi.org/10.1016/S0196-0 644 (00)70105-3

[15] Richards JO, Navarro MI, Derlet RO. Survey of directors of emergency departments in California on overcrowding West J Med. 2000; 172(6): 385-8. PMid: 10854386. http://dx.doi.org/10.1136 /ewjm.172.6.385

[16] Bullard MJ, Villa-Roel C, Bond K. Tracking emergency department overcrowding in a tertiary care academic institution. Healthc Q. 2009; 12: 99-106. PMid: 19553772. http://dx.doi.org/10.12927/ hcq. 2013. 20884

[17] National Trauma Data Bank 2014 Annual Report [Internet]. Chicago, IL: American College of Surgeons; 2014. [cited 2015 May 5]. Available from: https: //www . facs.org/ /media/files/quality\%20programs /trauma/ntdb/ntdb\%20annual\%20report\%202014.ashx 